\title{
ONE-COMPLEMENTED SUBSPACES OF REAL SEQUENCE SPACES
}

\author{
BEATA RANDRIANANTOANINA
}

\begin{abstract}
Characterizations are given for 1-complemented hyperplanes of strictly monotone real Lorentz spaces and 1-complemented finite codimensional subspaces (which contain at least one basis element) of real Orlicz spaces equipped with either Luxemburg or Orlicz norm.
\end{abstract}

\section{INTRODUCTION}

The question about the form of projections and the structure of their ranges arises naturally in geometry of Banach spaces and has many applications in other fields. Thus it has been studied by many authors since the time of Banach, but still even the structure of norm-one projections and 1-complemented subspaces is far from understood for most Banach spaces (for more detailed discussions of applications and some of the open questions see, e.g., the surveys [5, 6]).

In this paper we study which subspaces of finite codimension are 1-complemented in real Orlicz and Lorentz sequence spaces. In the earlier paper [18], the author gave necessary conditions for 1-complementability of subspaces of complex spaces with 1-unconditional bases. However, the methods of the complex case do not transfer to the real case. The main difference is that every 1-complemented subspace of a complex space with 1-unconditional basis also has a 1-unconditional basis ([10, 7), but this fact fails for subspaces of real spaces ([11, 2]), and even for subspaces of symmetric real spaces (since every space with 1-unconditional basis is 1-complemented in some symmetric space [12], [13, Theorem 3.b.1]).

Among the real sequence spaces, only 1-complemented subspaces of $\ell_{p}, 1 \leq p \leq \infty$ are fully understood. Namely, it is well known that a subspace $Y \subset \ell_{p},(1 \leq p<\infty, p \neq 2)$ is 1-complemented if and only if $Y$ is spanned by a block basis of a permutation of the original basis ([13, Theorem 2.a.4]). The only other available description is a sufficient condition for 1-complementability of subspaces of symmetric sequence spaces; namely subspaces spanned by a block basis with constant coefficients of a permutation of the original basis are 1complemented in symmetric spaces ([13, Proposition 3.a.4]).

1991 Mathematics Subject Classification. 46B,46E. 
In the present paper, we show that the sufficient condition above is also necessary for 1-complementability of:

1. hyperplanes in strictly monotone Lorentz spaces not isometric to $\ell_{p}, 1 \leq p<\infty$, (Corollary 6), and

2. finite codimensional subspaces containing at least one basis element of Orlicz spaces (with either Luxemburg or Orlicz norm) not isomorphic to any $\ell_{p}, 1 \leq p<\infty$ (Corollary 10).

We use a technique of numerically positive operators (defined by formula (11) below), and our main tool is that a projection $P$ has norm one if and only if $(I d-P)$ is numerically positive (Proposition 11).

We use standard Banach space notation; all undefined terms may be found, for example, in 13 .

\section{Preliminaries}

Throughout the paper we will consider a real Banach space $X$ with 1-unconditional basis $\left\{e_{i}\right\}_{i \in I}$. Most often $X$ will be either a Lorentz or an Orlicz sequence space. We recall their definitions below (see [13]).

Let $1 \leq p<\infty$ and let $w=\left(w_{n}\right)_{n}$ be a non-increasing sequence of nonnegative numbers with $w_{1}=1$. Then the Lorentz space $\ell_{w, p}$ is a space of all sequences $x=\left(x_{n}\right)_{n}$ for which $\|x\|_{w, p}<\infty$ where

$$
\|x\|_{w, p}=\left(\sum_{n=1}^{\operatorname{dim} \ell_{w, p}} w_{n}\left(x_{n}^{*}\right)^{p}\right)^{\frac{1}{p}}
$$

and where $\left(x_{n}^{*}\right)_{n}$ is the non-increasing rearrangement of $\left(\left|x_{n}\right|\right)_{n}$.

A function $\varphi:[0, \infty) \longrightarrow[0, \infty)$ is called an Orlicz function if $\varphi$ is non-decreasing, convex and $\varphi(1)=1$. An Orlicz space $\ell_{\varphi}$ is a space of sequences $x=\left(x_{n}\right)_{n=1}^{\operatorname{dim} \ell_{\varphi}}$ such that there exists $\lambda>0$ with

$$
\sum_{n=1}^{\operatorname{dim} \ell_{\varphi}} \varphi\left(\frac{\left|x_{n}\right|}{\lambda}\right)<\infty .
$$

It is customary to consider two (equivalent) norms on $\ell_{\varphi}$ : the Luxemburg and the Orlicz norm. The Luxemburg norm is given by

$$
\|x\|_{\varphi}=\inf \left\{\lambda>0: \sum_{n=1}^{\operatorname{dim} \ell_{\varphi}} \varphi\left(\frac{\left|x_{n}\right|}{\lambda}\right) \leq 1\right\}
$$


and the Orlicz norm is given by

$$
\|x\|_{\varphi, O}=\sup \left\{\sum_{n=1}^{\operatorname{dim} \ell_{\varphi}} x_{n} y_{n}: \sum_{n=1}^{\operatorname{dim} \ell_{\varphi}} \varphi^{*}\left(\left|y_{n}\right|\right) \leq 1\right\}
$$

where $\varphi^{*}$ denotes the Orlicz function complementary to $\varphi$ in the sense of Young, i.e. for any $u \geq 0$

$$
\varphi^{*}(u)=\sup \{t u-\varphi(t): 0<t<\infty\}
$$

Let $X$ be a real Banach space. We say that a functional $x^{*} \in X^{*}$ is norming for $x \in X$ if and only if $\left\|x^{*}\right\|=\|x\|$ and $x^{*}(x)=\|x\|^{2}$. Denote $\Pi(X)=\left\{\left(x, x^{*}\right):\|x\|=\right.$ 1 and $x^{*}$ is norming for $\left.\mathrm{x}\right\} \subset\left(X, X^{*}\right)$. Following Rosenthal [20] we say that an operator $T: X \longrightarrow X$ is numerically positive if

$$
x^{*}(T x) \geq 0
$$

whenever $\left(x, x^{*}\right) \in \Pi(X)$. This is equivalent to requiring a slightly weaker condition that given $x$ with $\|x\|=1$ there exists $x^{*}$ so that $\left(x, x^{*}\right) \in \Pi(X)$ and $x^{*}(T x) \geq 0$ (see Lumer [15, 16]). By results of Lumer [15] and Lumer and Phillips [17] (see also [3]) it is equivalent to the requirement that $\|\exp (-\alpha T)\| \leq 1$ for $\alpha \geq 0$. This immediately implies the following simple fact, which is the main tool for our applications :

Proposition 1. (see [20, 9]) If $X$ is a real Banach space and $P: X \longrightarrow X$ is a projection then $P$ is numerically positive if and only if $\|I d-P\|=1$, where Id denotes the identity on $X$.

We need to introduce the following technical property. We say that a space $X$ with 1-unconditional basis $\left\{e_{i}\right\}_{i \in I}$ has property $(P)$ if for all $i, j \in I$ and for all $\varepsilon>0$

$$
\left\|e_{i}+\varepsilon e_{j}\right\|_{X}>\left\|e_{i}\right\|_{X}=1 .
$$

Notice that property $(P)$ is slightly weaker than strict monotonicity, e.g., a Lorentz sequence spaces $\ell_{w, p}$ satisfies $(P)$ whenever $w_{2} \neq 0$, where $w=\left(w_{1}, w_{2}, w_{3}, \ldots\right)$ for all $p, 1 \leq p<\infty$, i.e., $\ell_{w, p} \in(P)$ whenever $\ell_{w, p}$ is not isometric to $\ell_{\infty}$.

An Orlicz sequence space $\ell_{\varphi}$ satisfies $(P)$ whenever $\varphi(t)>0$ for all $t>0$ and $\varphi(1)=1$.

We say that a space $X$ with 1 -unconditional basis $\left\{e_{i}\right\}_{i \in I}$ has property $(Q)$ if for all $i, j \in I$

$$
\lim _{\varepsilon \rightarrow 0} \frac{\left\|e_{i}+\varepsilon e_{j}\right\|_{X}-1}{\varepsilon}=0
$$

Notice that a Lorentz sequence space $\ell_{w, p}$ satisfies $(Q)$ whenever $p>1$, and Orlicz sequence space $\ell_{\varphi}$ satisfies $(Q)$ whenever $\varphi^{\prime}(0)=0$. 
We will be interested in finite codimensional subspaces of real Banach spaces. Notice that a subspace $Y \subset X$, codim $Y=n$, is spanned by disjointly supported vectors if and only if $Y$ can be presented in the form $Y=\bigcap_{j=1}^{n}$ ker $g_{j}$, where functionals $g_{j}$ are such that card $\left(\operatorname{supp} g_{j}\right) \leq 2$ for all $j \leq n$. We will consider the "standard" form of $Y \subset X$, $\operatorname{codim} Y=n$, i.e., $Y=\bigcap_{j=1}^{n} \operatorname{ker} f_{j}$, where functionals $f_{j}$ are such that $f_{j k}=\delta_{j k}$ for all $j, k \leq n$. Further, we will consider a projection $P: X \stackrel{\text { onto }}{\longrightarrow} Y$, where $P=I d-\sum_{j=1}^{n} f_{j} \otimes u_{j}$; here $I d$ denotes the identity on $X$ and $u_{1}, \ldots, u_{n}$ are linearly independent elements in $X$ with $f_{j}\left(u_{k}\right)=\delta_{j k}$. In this notation we have:

Lemma 2. Suppose that $X$ satisfies properties $(P)$ and $(Q)$ and that the projection $P: X \rightarrow$ $Y$ has norm one. Then

$$
\bigcup_{j=1}^{n} \operatorname{supp} u_{j}=\bigcup_{j=1}^{n} \operatorname{supp} f_{j} \text {. }
$$

Proof. If $k \notin \bigcup_{j=1}^{n} \operatorname{supp} u_{j}$ then

$$
\left(P\left(e_{k}\right)\right)_{k}=1-\sum_{j=1}^{n} f_{j}\left(e_{k}\right) u_{j k}=1
$$

Since $\|P\|=1$, property $(P)$ implies that $P\left(e_{k}\right)=e_{k}$. Hence $e_{k} \in \bigcap_{j=1}^{n} \operatorname{ker} f_{j}=Y$ and $k \notin \bigcup_{j=1}^{n} \operatorname{supp} f_{j}$. Therefore $\bigcup_{j=1}^{n} \operatorname{supp} f_{j} \subset \bigcup_{j=1}^{n} \operatorname{supp} u_{j}$.

For the other inclusion, consider $k \notin \bigcup_{j=1}^{n} \operatorname{supp} f_{j}$. Let $M=\max \left\{\left|u_{j j}\right|: j=1,2, \ldots, n\right\}+$ 1. Fix arbitrary $i, 1 \leq i \leq n, \varepsilon \neq 0$, and consider element $x_{\varepsilon}=e_{k}+\varepsilon e_{i}$. Denote by $x_{\varepsilon}^{N}=a_{\varepsilon} e_{k}^{*}+b_{\varepsilon} e_{i}^{*}$ a norming element for $x_{\varepsilon}$ with $\left\|x_{\varepsilon}^{N}\right\|_{X^{*}}=1$. By property $(P)$ of $X b_{\varepsilon} \neq 0$ whenever $\varepsilon \neq 0$. Since, by Proposition 11, $\sum_{j=1}^{n} f_{j} \otimes u_{j}$ is numerically positive, we get for all $\varepsilon \neq 0$ :

$$
\begin{aligned}
0 & \leq \sum_{j=1}^{n} f_{j}\left(x_{\varepsilon}\right) x_{\varepsilon}^{N}\left(u_{j}\right)=\sum_{j=1}^{n}\left(f_{j k}+\varepsilon f_{j i}\right)\left(a_{\varepsilon} u_{j k}+b_{\varepsilon} u_{j i}\right) \\
& =\sum_{j=1}^{n} \varepsilon \delta_{j i}\left(a_{\varepsilon} u_{j k}+b_{\varepsilon} u_{j i}\right)=\varepsilon\left(a_{\varepsilon} u_{i k}+b_{\varepsilon} u_{i i}\right) .
\end{aligned}
$$

Thus for all $\varepsilon \neq 0$

$$
\operatorname{sgn}\left(a_{\varepsilon} u_{i k}+b_{\varepsilon} u_{i i}\right)=\operatorname{sgn} \varepsilon=\operatorname{sgn}\left(b_{\varepsilon}\right) .
$$

By property $(Q)$ of $X \lim _{\varepsilon \rightarrow 0} b_{\varepsilon}=0$. Thus (2) implies that $u_{i k}=0$. Indeed, if $\left|u_{i k}\right|=\eta>0$ let $\varepsilon$ be small enough so that $\left|b_{\varepsilon}\right|<\frac{\eta}{2 M}$ and $a_{\varepsilon}>\frac{1}{2}$. Then

$$
\left|b_{\varepsilon} u_{i i}\right|<\frac{\eta}{2 M} \cdot M=\frac{\eta}{2}<a_{\varepsilon} \cdot\left|u_{i k}\right| .
$$

Thus $\operatorname{sgn}\left(a_{\varepsilon} u_{i k}+b_{\varepsilon} u_{i i}\right)=\operatorname{sgn}\left(a_{\varepsilon} u_{i k}\right)$ for all $\varepsilon$, contradicting (2). 


\section{LORENTZ SEQUenCE SPACES $\ell_{w, p}, p>1$}

In this section we study 1-complemented hyperplanes of $\ell_{w, p}$ where $p>1$.

Theorem 3. Let $\ell_{w, p}$ be a Lorentz sequence space with $1<p<\infty$ and $w_{2}>0$. Suppose that $Y=\operatorname{ker} f$ is 1-complemented in $\ell_{w, p}$ and $\operatorname{card}(\operatorname{supp} f) \geq n>2$. Then $p=2$ and $1=w_{1}=w_{2} \ldots=w_{n}$.

Our formulation resembles the characterizations of 1-complemented finite codimensional subspaces of $\ell_{p}$ in terms of their representation as an intersection of kernels of functionals $Y=\bigcap \operatorname{ker} f_{j}$ (see [1]).

We postpone the proof of Theorem 3 to the end of this section.

As a consequence of Theorem [3, we obtain the following characterization of $\ell_{2}$ among Lorentz sequence spaces:

Corollary 4. Let $\ell_{w, p}$ be a Lorentz sequence space with $1<p<\infty$ and $w_{i}>0$ for all $i \geq 1$. Suppose that there exists a functional $f$ such that $\operatorname{card}(\operatorname{supp} f)>2$ and $\operatorname{ker} f$ is 1-complemented in $\ell_{w, p}$. Then $\ell_{w, p}=\ell_{2}$.

In the above corollary, the assumption about nonzero weights is equivalent to asking that $\ell_{w, p}$ be strictly monotone. It can be slightly relaxed if $\operatorname{card}(\operatorname{supp} f)=\infty$ or if $\operatorname{dim} \ell_{w, p}<\infty$.

(a) If $\operatorname{card}(\operatorname{supp} f)=\infty$ it is enough to assume that $w_{2}>0$.

(b) If $\operatorname{dim} \ell_{w, p}=d<\infty$ and $\operatorname{card}(\operatorname{supp} f)=n \leq d$ it is enough to assume that $w_{d-n+3}>0$.

However the assumption about nonzero weights cannot be completely removed as the following example demonstrates.

Example . Let $w=(1,1,1,0)$ and consider a 4-dimensional space $\ell_{w, 2}^{4}$. Let $Y=\operatorname{ker}\left(e_{1}^{*}+\right.$ $\left.e_{2}^{*}+e_{3}^{*}\right)$ and $P_{2}: \ell_{2}^{3} \longrightarrow \ell_{2}^{3}$ be the orthogonal projection. Define $P: \ell_{w, 2}^{4} \longrightarrow \ell_{w, 2}^{4}$ by

$$
P\left(x_{1}, x_{2}, x_{3}, x_{4}\right)=P_{2}\left(x_{1}, x_{2}, x_{3}\right)+0 \cdot e_{4} .
$$

Then:

$$
\left\|P\left(x_{1}, x_{2}, x_{3}, x_{4}\right)\right\|_{w, 2}=\left\|P_{2}\left(x_{1}, x_{2}, x_{3}\right)\right\|_{2} \leq\left\|\left(x_{1}, x_{2}, x_{3}, 0\right)\right\|_{2} \leq\left\|\left(x_{1}, x_{2}, x_{3}, x_{4}\right)\right\|_{w, 2} .
$$

Thus $\|P\| \leq 1$ i.e. $\operatorname{ker}\left(e_{1}^{*}+e_{2}^{*}+e_{3}^{*}\right)$ is 1-complemented in $\ell_{w, 2}^{4} \neq \ell_{2}^{4}$.

The condition $\operatorname{card}(\operatorname{supp} f) \leq 2$ means that $\operatorname{ker} f$ is spanned by disjointly supported vectors. In [18] the author studied the form of 1-complemented disjointly spanned subspaces of Lorentz spaces and she obtained: 
Theorem 5. ([18, Theorem 6.3]) Let $\ell_{w, p}$, with $1<p<\infty$, be a real or complex Lorentz sequence space. Suppose that $\left\{x_{i}\right\}_{i \in I}$ are mutually disjoint elements of $\ell_{w, p}$ such that $\operatorname{card}(I) \geq$ 2 and $F=\overline{\operatorname{span}}\left\{x_{i}\right\}_{i \in I}$ is 1-complemented in $\ell_{w, p}$. Suppose, moreover, that $w_{\nu} \neq 0$ for all $\nu \leq \Sigma \stackrel{\text { def }}{=} \sum_{i \in I} \operatorname{card}\left(\operatorname{supp} x_{i}\right) \quad(\leq \infty)$.

Then

(a) $w_{\nu}=1$ for all $\nu \leq \Sigma$,

or

(b) $\left|x_{i l}\right|=\left|x_{i k}\right|$ for all $i \in I$ and all $k, l \in \operatorname{supp} x_{i}$.

Thus, as an immediate consequence of Theorem 3 and [18, Theorem 6.3], we get:

Corollary 6. Let $\ell_{w, p}$ be a Lorentz sequence space with $1<p<\infty$ and $w_{k}>0$ for all $k$, i.e. $\ell_{w, p}$ is strictly monotone. Suppose that $Y=\operatorname{ker} f$ is 1 -complemented in $\ell_{w, p}$ and $\operatorname{card}(\operatorname{supp} f)=2$, i.e. $f=f_{i} e_{i}{ }^{*}+f_{j} e_{j}{ }^{*}$ for some $i \neq j$.

Then $\left|f_{i}\right|=\left|f_{j}\right|$ for all $i, j$, or $\ell_{w, p}=\ell_{p}$, i.e. $w_{k}=1$ for all $k$.

Proof of Corollary 4 . If $2<\operatorname{card}(\operatorname{supp} f)=\operatorname{dim} \ell_{w, p} \leq \infty$ then the corollary follows immediately from Theorem 3 .

Assume thus, that $2<\operatorname{card}(\operatorname{supp} f)=n<\operatorname{dim} \ell_{w, p} \leq \infty$. We will prove by induction that $w_{m}=w_{1}=1$ for all $m \leq \operatorname{dim} \ell_{w, p}$. The first induction step follows from Theorem 3 , also we get that $p=2$.

Assume now that $w_{m}=1$ for some $n \leq m<\operatorname{dim} \ell_{w, p}$. We will show that $w_{m+1}=1$.

Let $\left\{i_{1}, \ldots, i_{m-n+1}\right\} \subset\left\{1, \ldots, \operatorname{dim} \ell_{w, 2}\right\}$ be such that $\left\{i_{1}, \ldots, i_{m-n+1}\right\} \cap \operatorname{supp} f=\emptyset$.

Consider a Lorentz space $\ell_{w^{\prime}, 2}=\operatorname{span}\left\{e_{i}: i \notin\left\{i_{1}, \ldots, i_{m-n+1}\right\}\right\}$, where $w^{\prime}$ is a weight defined by $w_{k}^{\prime}=w_{k+m-n+1}$.

By inductive hypothesis, $w_{1}^{\prime}=\ldots=w_{n-1}^{\prime}=w_{m}=1$.

For any $x \in \ell_{w, p}$ with supp $x \subseteq \operatorname{supp} f$, consider an element

$$
x_{+} \stackrel{\text { def }}{=} x+\sum_{k=1}^{m-n+1}\|x\|_{w, 2} e_{i_{k}}
$$

Since $\left\{e_{i_{1}}, \ldots, e_{i_{m-n+1}}\right\} \subset \operatorname{ker} f$, we have

$$
P x_{+}=P x+\sum_{k=1}^{m-n+1}\|x\|_{w, 2} P e_{i_{k}}=P x+\sum_{k=1}^{m-n+1}\|x\|_{w, 2} e_{i_{k}} .
$$

Since $P$ is a contractive projection $\|P x\|_{w, 2} \leq\|x\|_{w, 2}$, and thus $\|x\|_{w, 2} \geq\left|(P x)_{i}\right|$ for all $i \in \operatorname{supp} x$. Clearly also $\|x\|_{w, 2} \geq\left|x_{i}\right|$ for all $i \in \operatorname{supp} x$. Hence

$$
\left\|x_{+}\right\|_{w, 2}^{2}=\sum_{k=1}^{m-n+1}\|x\|_{w, 2}^{2}+\|x\|_{w^{\prime}, 2}^{2}
$$


and

$$
\left\|P x_{+}\right\|_{w, 2}^{2}=\sum_{k=1}^{m-n+1}\|x\|_{w, 2}^{2}+\|P x\|_{w^{\prime}, 2}^{2} .
$$

Since $P$ is contractive in $\ell_{w, 2}$ we conclude that $\|P x\|_{w^{\prime}, 2} \leq\|x\|_{w^{\prime}, 2}$ for all $x$ with $\operatorname{supp} x \cap$ $\left\{i_{1}, \ldots, i_{m-n+1}\right\}=\emptyset$. That is, $P$ is contractive in $\ell_{w^{\prime}, 2}$. Thus ker $f$ is 1 -complemented in $\ell_{w^{\prime}, 2}$ and, by Theorem 3, since $\operatorname{card}(\operatorname{supp} f)=n$ and $w_{2}^{\prime}=w_{m-n+3} \geq w_{m}>0$, we get $w_{n}^{\prime}=1$. Thus $w_{m+1}=1$, as claimed.

Proof of Theorem 3. Since $\ell_{w, p}$ is symmetric we can assume without loss of generality that

$$
f=\left(f_{1}, f_{2}, f_{3}, \ldots, f_{n}, \ldots\right)
$$

where $f_{1} \geq f_{2} \geq \cdots \geq f_{n}>0$. Then $f \otimes u$ is numerically positive $\left(u=\left(u_{1}, u_{2}, \ldots, u_{n}, \ldots\right)\right)$ and, by Lemma 2, $u_{1}, u_{2}, \ldots, u_{n} \neq 0$.

We organize the proof of Theorem 3 into four assertions:

Assertion 1: If $n \geq 4$ or $n=3$ and $f_{3}<f_{1}$ then $p=2$ and

$$
\begin{gathered}
\frac{w_{2}+\ldots+w_{n-1}}{n-2} \sum_{i=1}^{n-2} u_{i}=\frac{f_{1}+\ldots+f_{n-2}}{f_{n}} u_{n} \\
u_{n}=\frac{f_{n}}{f_{n-1}} w_{n} u_{n-1}
\end{gathered}
$$

Assertion 2: If $n \geq 4$ or $\left(n=3\right.$ and $\left.f_{2}<f_{1}\right)$ then $p=2$ and $w_{n}=1$, i.e. Theorem 3 holds.

Assertion 3: If $n=3$ and $f_{1}=f_{2}$ then $p=2, w_{2}=1$ and

$$
u_{1}=\frac{f_{1}}{f_{3}} w_{3} u_{3}
$$

Assertion 4: If $n=3$ then $p=2$ and $w_{3}=1$, i.e. Theorem 3 holds.

In the proof of all assertions, we will use the following elements $x(a)$ and $x(a, \varepsilon)$ :

$$
\begin{aligned}
& x(a)=e_{1}+\ldots+e_{n-2}-\frac{f_{1}+\ldots+f_{n-2}}{f_{n-1}} a e_{n-1}-\frac{f_{1}+\ldots+f_{n-2}}{f_{n}}(1-a) e_{n} \\
x(a, \varepsilon)= & x(a)-\varepsilon e_{n-1}-\varepsilon e_{n} \\
= & e_{1}+\ldots+e_{n-2}-\left(\frac{f_{1}+\ldots+f_{n-2}}{f_{n-1}} a+\varepsilon\right) e_{n-1}-\left(\frac{f_{1}+\ldots+f_{n-2}}{f_{n}}(1-a)+\varepsilon\right) e_{n},
\end{aligned}
$$

where $a \in[0,1], \varepsilon \in[-1,1]$.

Notice that for all $a, x(a) \in \operatorname{ker} f$.

For the proof of Assertion 1, let

$$
\eta=\min \left(\frac{f_{n-1}}{f_{1}+\ldots+f_{n-2}}, 1-\frac{f_{n}}{f_{1}+\ldots+f_{n-2}}\right) .
$$


Notice that by assumptions of Assertion 1, $\eta>0$.

For any $a$ with $0<a<\eta$ we have

$$
\frac{f_{1}+\ldots+f_{n-2}}{f_{n}}(1-a)>1>\frac{f_{1}+\ldots+f_{n-2}}{f_{n-1}}>0 .
$$

For any $a$ with $0<a<\eta$ define

$$
\delta(a)=\min \left(\frac{f_{1}+\ldots+f_{n-2}}{f_{n}}(1-a)-1,1-\frac{f_{1}+\ldots+f_{n-2}}{f_{n-1}}, \frac{f_{1}+\ldots+f_{n-2}}{f_{n-1}}\right) .
$$

Then, for any $\varepsilon$ with $|\varepsilon|<\delta(a)$

$$
\left|x(a, \varepsilon)_{n}\right|>\left|x(a, \varepsilon)_{1}\right|=\ldots=\left|x(a, \varepsilon)_{n-2}\right|>\left|x(a, \varepsilon)_{n-1}\right| .
$$

Thus, a norming functional for $x(a, \varepsilon)$ can be chosen as follows:

$$
\begin{aligned}
x(a, \varepsilon)^{N}= & \sum_{i=1}^{n-2}\left(\frac{w_{2}+\ldots+w_{n-1}}{n-2}\right) e_{i}^{*}-\left(\frac{f_{1}+\ldots+f_{n-2}}{f_{n-1}} a+\varepsilon\right)^{p-1} w_{n} e_{n-1}^{*} \\
& -\left(\frac{f_{1}+\ldots+f_{n-2}}{f_{n}}(1-a)+\varepsilon\right)^{p-1} e_{n}^{*} .
\end{aligned}
$$

Since $f \otimes u$ is numerically positive, for all $a, 0<a<\eta$ and $\varepsilon$ with $|\varepsilon|<\delta(a)$ we have

$$
f(x(a, \varepsilon)) x(a, \varepsilon)^{N}(u) \geq 0 .
$$

Hence:

$$
\begin{gathered}
f(x(a, \varepsilon))=f(x(a))-\varepsilon f\left(e_{n-1}+e_{n}\right) \\
=0-\varepsilon\left(f_{n-1}+f_{n}\right)=-\varepsilon\left(f_{n-1}+f_{n}\right) . \\
x(a, \varepsilon)^{N}(u)=\frac{w_{2}+\ldots+w_{n-1}}{n-2} \sum_{i=1}^{n-2} u_{i}-\left(\frac{f_{1}+\ldots+f_{n-2}}{f_{n-1}} a+\varepsilon\right)^{p-1} w_{n} u_{n-1} \\
-\left(\frac{f_{1}+\ldots+f_{n-2}}{f_{n}}(1-a)+\varepsilon\right)^{p-1} u_{n} .
\end{gathered}
$$

Thus

$$
-\operatorname{sgn} \varepsilon=\operatorname{sgn}(f(x(a, \varepsilon)))=\operatorname{sgn}\left(x(a, \varepsilon)^{N}(u)\right) .
$$

Since $p>1$ we conclude that

$$
x(a, \varepsilon)^{N}(u)=0 .
$$


Hence:

$$
\begin{aligned}
\frac{w_{2}+\ldots+w_{n-1}}{n-2} \sum_{i=1}^{n-2} u_{i}-\left(\frac{f_{1}+\ldots+f_{n-2}}{f_{n-1}}\right)^{p-1} w_{n} u_{n-1} a^{p-1} & \\
& -\left(\frac{f_{1}+\ldots+f_{n-2}}{f_{n}}\right)^{p-1} u_{n}(1-a)^{p-1}=0 .
\end{aligned}
$$

Notice that (6) is valid for all $a$ with $0<a<\eta$ and all parameters in (6) except $a$ are fixed, i.e. (6) in fact is:

$$
A+B a^{p-1}+C(1-a)^{p-1}=0
$$

where $A, B, C$ are constants such that $A, C \neq 0$; and $B=0$ if and only if $w_{n}=0$.

If $B=0$ we get

$$
(1-a)^{p-1}=\left(-\frac{A}{C}\right)=\text { const. }
$$

for all $a \in(0, \eta)$, which is impossible since $p>1$.

If $B \neq 0$ we differentiate and get

$$
\left(\frac{a}{1-a}\right)^{p-2}=-\frac{C}{B}=\text { const. }
$$

Thus $p=2$.

When $p=2$, equation (6) becomes

$$
\begin{aligned}
& \left(\frac{w_{2}+\ldots+w_{n-1}}{n-2} \sum_{i=1}^{n-2} u_{i}-\frac{f_{1}+\ldots+f_{n-2}}{f_{n}} u_{n}\right) \\
& +a\left(\frac{f_{1}+\ldots+f_{n-2}}{f_{n}} u_{n}-\frac{f_{1}+\ldots+f_{n-2}}{f_{n-1}} w_{n} u_{n-1}\right)=0
\end{aligned}
$$

for all $a \in(0, \eta)$.

Thus

$$
\frac{w_{2}+\ldots+w_{n-1}}{n-2} \sum_{i=1}^{n-2} u_{i}=\frac{f_{1}+\ldots+f_{n-2}}{f_{n}} u_{n}
$$

and

$$
u_{n}=\frac{f_{n}}{f_{n-1}} w_{n} u_{n-1} .
$$

This finishes the proof of Assertion 1.

For the proof of Assertion 2, assume that $n \geq 4$ or that $\left(n=3\right.$ and $f_{2}<f_{1}$ ).

Notice first that the assumptions of Assertion 2 are stronger than those of Assertion 1. Thus, we have $p=2$. 
Consider elements $x(a, \varepsilon)$ with $a=1$ and

$$
|\varepsilon|<\varepsilon_{1} \stackrel{\text { def }}{=} \min \left(\frac{f_{1}+\ldots+f_{n-2}}{f_{n-1}}-1,1\right) \text {. }
$$

Notice that by our assumptions, $\varepsilon_{1}>0$.

Then

$$
x(1, \varepsilon)=e_{1}+\ldots+e_{n-2}-\left(\frac{f_{1}+\ldots+f_{n-2}}{f_{n-1}}+\varepsilon\right) e_{n-1}-\varepsilon e_{n}
$$

and

$$
\left|x(1, \varepsilon)_{n-1}\right|>\left|x(1, \varepsilon)_{1}\right|=\ldots=\left|x(1, \varepsilon)_{n-2}\right|>\left|x(1, \varepsilon)_{n}\right| .
$$

Thus a norming functional for $x(1, \varepsilon)$ can be chosen to be

$$
x(1, \varepsilon)^{N}=\left(\frac{w_{2}+\ldots+w_{n-1}}{n-2}\right) \sum_{i=1}^{n-2} e_{i}^{*}-\left(\frac{f_{1}+\ldots+f_{n-2}}{f_{n-1}}+\varepsilon\right) e_{n-1}^{*}-\varepsilon w_{n} e_{n}{ }^{*} .
$$

Similarly as in (5) we conclude that

$$
x(1,0)^{N}(u)=0 .
$$

Hence

$$
\frac{w_{2}+\ldots+w_{n-1}}{n-2} \sum_{i=1}^{n-2} u_{i}=\frac{f_{1}+\ldots+f_{n-2}}{f_{n-1}} u_{n-1} .
$$

Combining this with (7) we get

$$
u_{n}=u_{n-1}
$$

and further, by (8),

But $f_{n} \leq f_{n-1}$, thus

$$
\frac{f_{n}}{f_{n-1}} w_{n}=1
$$

Hence $w_{n}=1$ and Assertion 2 is proved.

For the proof of Assertion 3, assume that $n=3$ and $f_{1}=f_{2}$.

By symmetry of $\ell_{w, p}$ we can assume without loss of generality that $u_{1}=u_{2}$. Similarly as in previous cases, consider the element $x(a, \varepsilon)$ with $a \in\left(f_{2} /\left(f_{2}+f_{3}\right), 1\right)$ and

$$
|\varepsilon|<\varepsilon(a) \stackrel{\text { def }}{=} \min \left(1-a, \frac{1}{2}\left(a-\frac{f_{1}}{f_{3}}(1-a)\right)\right) .
$$

Notice that $\varepsilon(a)>0$ when $a \in\left(f_{2} /\left(f_{2}+f_{3}\right), 1\right)$. Then

$$
x(a, \varepsilon)=e_{1}-(a+\varepsilon) e_{2}-\left(\frac{f_{1}}{f_{3}}(1-a)+\varepsilon\right) e_{3}
$$


and

$$
\left|x(a, \varepsilon)_{1}\right|>\left|x(a, \varepsilon)_{2}\right|>\left|x(a, \varepsilon)_{3}\right| .
$$

Thus the norming functional for $x(a, \varepsilon)$ is given by

$$
x(a, \varepsilon)^{N}=e_{1}{ }^{*}-(a+\varepsilon)^{p-1} w_{2} e_{2}{ }^{*}-\left(\frac{f_{1}}{f_{3}}(1-a)+\varepsilon\right)^{p-1} w_{3} e_{3}{ }^{*} .
$$

Similarly as in (5), we conclude that

$$
x(a, 0)^{N}(u)=0 .
$$

Thus, for all $a \in\left(f_{2} /\left(f_{2}+f_{3}\right), 1\right)$,

$$
u_{1}-a^{p-1} w_{2} u_{2}-\left(\frac{f_{1}}{f_{3}}(1-a)\right)^{p-1} w_{3} u_{3}=0 .
$$

By the same argument as in (6), we see that $p=2$; and (9) becomes

$$
u_{1}-\frac{f_{1}}{f_{3}} w_{3} u_{3}+a\left(\frac{f_{1}}{f_{3}} w_{3} u_{3}-w_{2} u_{2}\right)=0 .
$$

Since this equality holds for all $a \in\left(f_{2} /\left(f_{2}+f_{3}\right), 1\right)$ we have

$$
u_{1}=\frac{f_{1}}{f_{3}} w_{3} u_{3}
$$

and

$$
\frac{f_{1}}{f_{3}} w_{3} u_{3}=w_{2} u_{2} .
$$

Since $u_{1}=u_{2}$ we conclude that $w_{2}=1$, which ends the proof of Assertion 3 .

Finally, for the proof of Assertion 4, assume that $n=3$.

If $f_{2}<f_{1}$ then by Assertion 2, Theorem 3 holds.

If $f_{2}=f_{1}$ and $f_{3}<f_{1}$ then $u_{1}=u_{2}$ and by Assertion $3, p=2, w_{2}=1$ and

$$
u_{1}=\frac{f_{1}}{f_{3}} w_{3} u_{3} .
$$

On the other hand, by Assertion 1,

$$
u_{3}=\frac{f_{3}}{f_{2}} w_{3} u_{2}
$$

Thus

$$
u_{1}=\frac{f_{1}}{f_{3}} w_{3} \frac{f_{3}}{f_{2}} w_{3} u_{2}=w_{3}^{2} u_{1}
$$

Hence $w_{3}=1$ and Theorem 3 holds.

If $f_{1}=f_{2}=f_{3}$ then by symmetry of $\ell_{w, p}, u_{1}=u_{2}=u_{3}$ and by Assertion 3 ,

$$
u_{1}=\frac{f_{1}}{f_{3}} w_{3} u_{3}=w_{3} u_{3}=w_{3} u_{1} .
$$


Thus $w_{3}=1$ and Theorem 3 holds.

\section{One-COMPlemented subspaces of Orlicz SEquence SPACES}

We say that $\varphi$ is similar to $t^{p}$ for some $p \in[1, \infty)$ if there exist $C, t_{0}>0$ so that $\varphi(t)=C t^{p}$ for all $t<t_{0}$. We say that $\varphi$ is equivalent to $t^{p}$ for some $p \in[1, \infty)$ if there exist $C_{1}, C_{2}, t_{0}>0$ so that $C_{1} t^{p} \leq \varphi(t) \leq C_{2} t^{p}$ for all $t<t_{0}$.

The main result of this section is the following:

Theorem 7. Let $\ell_{\varphi}$ be an Orlicz space such that $\varphi$ is not similar to $t^{2}$ and $\varphi(t)>0$ for all $t>0$. Consider $\ell_{\varphi}$ with either Luxemburg or Orlicz norm. Let $F \subset \ell_{\varphi}$ be a subspace of codimension $n$ with $\operatorname{dim} F>1$. If $F$ contains at least one basis vector and $F$ is 1 complemented in $\ell_{\varphi}$ then $F$ can be represented as $F=\bigcap_{j=1}^{n} \operatorname{ker} f_{j}$ where card $\left(\operatorname{supp} f_{j}\right) \leq 2$ for all $j$.

Moreover, if $\varphi$ is not equivalent to $t^{p}$ for any $p \in[1, \infty)$, then $\left|f_{j i}\right|$ is either 1 or 0 for all $i, j$.

If $\varphi$ is equivalent but not similar to $t^{p}$ for some $p \in[1, \infty)$, then there exists $\gamma \geq 1$ such that

$$
\left\{\left|f_{j i}\right|: j \leq n, i \leq \operatorname{dim} \ell_{\varphi}\right\} \subset\left\{\gamma^{m}: m \in \mathbb{Z}\right\} \cup\{0\}
$$

Similarly as in the case of Lorentz spaces our formulation resembles the characterizations of 1-complemented finite codimensional subspaces of $\ell_{p}$ in terms of their representation as an intersection of kernels of functionals $Y=\bigcap \operatorname{ker} f_{j}$ (see [1]).

As an immediate consequence of Theorem 7 we obtain the following:

Corollary 8. Let $\ell_{\varphi}$ be an Orlicz space with either Luxemburg or Orlicz norm, such that $\varphi$ is not equivalent to $t^{p}$ for any $1 \leq p \leq \infty$. Let $F \subset \ell_{\varphi}$ be a subspace of codimension $n$ with $\operatorname{dim} F>1$ and containing at least one basis vector. Then $F$ is 1 -complemented in $\ell_{\varphi}$ if and only if $F$ is a span of a block basis with constant coefficients.

Before the proof of Theorem 17 we note that the assumptions that $\varphi$ is is not similar to $t^{2}$ and $\varphi(t)>0$ for all $t>0$ cannot be removed. Indeed in [18] we presented an example of an Orlicz function $\varphi$ with $\varphi(t)=t^{2}$ for all $t \leq a$, where $\sqrt{2 / 3}<a<1$, and such that $\operatorname{ker}\left(e_{1}^{*}+e_{2}^{*}+e_{3}^{*}\right)$ is 1 -complemented in $\ell_{\varphi}$ ([18, Example 3]). This example can be easily modified so that for arbitrarily small $a>0$ we have $\varphi(t)=t^{2}$ when $t \leq a$, and $\ell_{\varphi}$ contains a 1-complemented subspace of codimension $n$ which cannot be represented as a span of disjointly supported vectors. 
Example . Let $\varphi$ be an Orlicz function such that $\varphi(t)=t^{2}$ for all $t$ with $0 \leq t \leq a$ for some $a>0$. Let $m \in \mathbb{N}$ be such that $1 / m \leq a^{2}$. Consider $\ell_{\varphi}^{3 m}$ and a subspace $F \subset \ell_{\varphi}^{3 m}$ defined by:

$$
F=\left\{\left(x_{i}\right)_{i=1}^{3 m}: x_{1}+x_{2}+x_{3}=0, x_{k}=x_{k+3 j}, k=1,2,3, j=1, \ldots, m-1\right\} .
$$

Then $F$ cannot be presented in the form described in the conclusion of Theorem 7 , but $F$ is 1-complemented in $\ell_{\varphi}^{3 m}$.

Note first that if $x \in F$ then $\|x\|_{\varphi}=\|x\|_{2}$. Indeed:

$$
1=\sum_{i=1}^{3 m} \varphi\left(\frac{\left|x_{i}\right|}{\|x\|_{\varphi}}\right)=m \sum_{k=1}^{3} \varphi\left(\frac{\left|x_{k}\right|}{\|x\|_{\varphi}}\right) .
$$

Thus for all $i \leq 3 m$ we have:

$$
\varphi\left(\frac{\left|x_{i}\right|}{\|x\|_{\varphi}}\right) \leq \frac{1}{m} \leq a^{2}
$$

Since $\varphi$ is increasing

$$
\varphi\left(\frac{\left|x_{i}\right|}{\|x\|_{\varphi}}\right)=\frac{\left|x_{i}\right|^{2}}{\|x\|_{\varphi}^{2}}
$$

for all $i \leq 3 m$ i.e. $\|x\|_{\varphi}=\|x\|_{2}$.

Let $Q: \ell_{2}^{3 m} \longrightarrow F$ be a contractive projection of $\ell_{2}^{3 m}$ onto $F$. Then $Q$ is also contractive when considered as a projection from $\ell_{\varphi}^{3 m}$ onto $F$. Indeed for all $x \in \ell_{\varphi}^{3 m}$ we have:

$$
\|x\|_{\varphi} \geq\|x\|_{2} \geq\|Q x\|_{2}=\|Q x\|_{\varphi}
$$

We were unable to eliminate the condition that subspace $F$ contains at least one basis vector in the assumptions of Theorem 47. However, it follows from the author's earlier work that this condition is satisfied in Orlicz spaces which are either $p$-convex with constant 1 for some $2<p<\infty$, or $q$-concave with constant 1 for some $1<q<2$ and $\varphi$ is smooth at 1 , provided that $\operatorname{dim} F<1 / 2 \operatorname{dim} X$. Namely, we have:

Theorem 9. ([19, Theorem 1]) Let $X$ be a strictly monotone sequence space $(\operatorname{dim} X=d \geq$ 3) with a 1-unconditional basis $\left\{e_{i}\right\}_{i=1}^{d}$. Suppose that

(a) $X$ is p-convex with constant $1,2<p<\infty$, or

(b) $X$ is $q$-concave with constant $1,1<q<2$, and smooth at each basic vector.

Then any 1-complemented subspace $F$ of codimension $n$ in $X$ contains all but at most $2 n$ basic vectors of $X$. 
Recall (see [14, Definition 1.d.3]) that a Banach space $X$ is $p$-convex with constant 1 (resp. $q$-concave with constant 1 ) if for every choice of elements $\left\{x_{i}\right\}_{i=1}^{n}$ in $X$ the following inequality holds:

$$
\left\|\left(\sum_{i=1}^{n}\left|x_{i}\right|^{p}\right)^{1 / p}\right\| \leq\left(\sum_{i=1}^{n}\left\|x_{i}\right\|^{p}\right)^{1 / p} \quad \text { if } 1 \leq p<\infty
$$

or, respectively,

$$
\left\|\left(\sum_{i=1}^{n}\left|x_{i}\right|^{q}\right)^{1 / q}\right\| \geq\left(\sum_{i=1}^{n}\left\|x_{i}\right\|^{q}\right)^{1 / q} \quad \text { if } 1 \leq q<\infty .
$$

Proof of Theorem 7 . We start with choosing a convenient notation. Let $F=\bigcap_{j=1}^{n} \operatorname{ker} f_{j}$ where $f_{j}$ 's are in the reduced form, i.e., $f_{j i}=\delta_{i j}$ for all $i, j \leq n$ and $f_{j, n+1}=0$ for all $j$. If $\operatorname{dim} \ell_{\varphi} \leq n+2$ there is nothing to prove so we will assume that $\operatorname{dim} \ell_{\varphi} \geq n+3$.

We claim that if there exist $j \leq n$ and $i, k>n$ with $f_{j i}, f_{j k} \neq 0$ then $\varphi$ is similar to $t^{2}$ or $\varphi(t) \ngtr 0$ for all $t>0$.

Assume, for contradiction, that $F$ is 1 -complemented and, say, $f_{1, n+2}, f_{1, n+3} \neq 0$. Then $P: \ell_{\varphi} \stackrel{\text { onto }}{\longrightarrow} F$ has form

$$
P(x)=x-\sum_{j=1}^{n} f_{j}(x) u_{j}
$$

where vectors $\left\{u_{j}\right\}_{j=1}^{n}$ are linearly independent. It is clear that we can select a subset $S^{\prime}$ of indices such that $\left\{\left.u_{j}\right|_{S^{\prime}}\right\}_{j=1}^{n}$ are linearly independent. Let $S=\{1,2, \ldots, n+3\} \cup S^{\prime}$. For convenience of notation we can assume without loss of generality that $S=\{1, \ldots, M\}$, where $M \leq \min \left\{\operatorname{dim} \ell_{\varphi}, 2 n+3\right\}$.

Let

$$
L=\left\|\sum_{j=1}^{n}\left(\sum_{i=n+2}^{M}\left|f_{j i}\right|+1\right) e_{j}+\sum_{j=n+2}^{M} e_{j}\right\|_{\varphi} .
$$

Here we denote by $\|\cdot\|_{\varphi}$ either the Luxemburg or Orlicz norm in $\ell_{\varphi}$.

For any $k \in\{1, \ldots, n\}, \varepsilon \in \mathbb{R}$ with $|\varepsilon|<1$ and any sequence $\left(\alpha_{j}\right)_{j=n+2}^{M} \subset[-1,1]$, consider elements $y$ and $y(k, \varepsilon)$ in $X$ defined by

$$
\begin{gathered}
y=\sum_{j=1}^{n}\left(-\sum_{i=n+2}^{M} f_{j i} \alpha_{i}\right) e_{j}+\sum_{j=n+2}^{M} \alpha_{j} e_{j} \in \bigcap_{j=1}^{n} \operatorname{ker} f_{j}, \\
y(k, \varepsilon)=y+\varepsilon e_{k} .
\end{gathered}
$$

Then $\|y(k, \varepsilon)\|_{\varphi} \leq L$. Thus there exists $\alpha_{k, \varepsilon}$ so that $\left\|y(k, \varepsilon)+\alpha_{(k, \varepsilon)} e_{n+1}\right\|_{\varphi}=L$. Set $x(k, \varepsilon)=$ $y(k, \varepsilon)+\alpha_{k, \varepsilon} e_{n+1}$ and $x=y(1,0)+\alpha_{1,0} e_{n+1}$. Then $x \in \bigcap_{j=1}^{n} \operatorname{ker} f_{j}$ and $f_{j}(x(k, \varepsilon))=\delta_{j k} \varepsilon$ for all $1 \leq k, j \leq n$. 
Since $I d-P=\sum_{j=1}^{n} f_{j} \otimes u_{j}$ is numerically positive, we conclude that

$$
\operatorname{sgn}\left(x(k, \varepsilon)^{N}\left(u_{k}\right)\right)=\operatorname{sgn} \varepsilon
$$

where $x(k, \varepsilon)^{N}$ denotes a norming functional for $x(k, \varepsilon)$. By [8] and [4 in both cases of Luxemburg and Orlicz norm on $\ell_{\varphi}$, a norming functional $x(k, \varepsilon)^{N}$ may be defined by the following formula:

$$
x(k, \varepsilon)^{N}{ }_{j}=\frac{1}{C} \operatorname{sgn}\left(x(k, \varepsilon)_{j}\right) \varphi^{\prime}\left(\frac{\left|x(k, \varepsilon)_{j}\right|}{L}\right) ; \quad j=1, \ldots, n
$$

Here $\varphi^{\prime}$ denotes the left derivative of $\varphi$ and $C>0$ is a constant depending on $x(k, \varepsilon)$, (recall that $L=\left\|x_{(k, \varepsilon)}\right\|_{\varphi}$ for all $(k, \varepsilon)$; of course $C$ depends also on the choice of either the Luxemburg or Orlicz norm).

Notice that for almost all $\left(\alpha_{j}\right)_{j=n+2}^{M} \in[-1,1]^{M-n-1}, \varphi^{\prime}$ is continuous at all points $\left\{\frac{\left|x_{(k, 0), j}\right|}{L}\right.$ : $j=1 \ldots M\}$. Denote the set of all such $\left(\alpha_{j}\right)_{j=n+2}^{M}$ by $\Lambda$. When $\left(\alpha_{j}\right)_{j=n+2}^{M} \in \Lambda$, since for every $k, x(k, 0)=x$, equation (11) implies that for all $k$ with $1 \leq k \leq n$

$$
x^{N}\left(u_{k}\right)=0,
$$

that is

$$
\sum_{j=1}^{n}-\operatorname{sgn}\left(\sum_{i=n+2}^{M} f_{j i} \alpha_{i}\right) \varphi^{\prime}\left(\frac{\left|\sum_{i=n+2}^{M} f_{j i} \alpha_{i}\right|}{L}\right) u_{k j}+\sum_{j=n+2}^{M} \operatorname{sgn}\left(\alpha_{j}\right) \varphi^{\prime}\left(\frac{\left|\alpha_{j}\right|}{L}\right) u_{k j}=0 .
$$

Thus, for any $(M-n-1)$-tuple $\left(\alpha_{j}\right)_{j=n+2}^{M}$ in $\Lambda$, (12) can be treated as an equation of variables $\left\{u_{k j}\right\}$ and it has $n$ linearly independent solutions:

$$
\left.u_{1}\right|_{S},\left.u_{2}\right|_{S}, \ldots,\left.u_{n}\right|_{S}
$$

Now we will look at equation (12) with specially selected $(M-n-1)$-tuples $\left(\alpha_{j}\right)_{j=n+2}^{M}$. For $r=1, \ldots, M-n-1$ consider $(M-n-1)$-tuples $\left(\alpha_{j}^{r}\right)_{j=n+2}^{M} \in \Lambda$ such that

$$
\alpha_{j}^{r}= \begin{cases}0 & \text { when } \quad j \neq n+r \\ \delta & \text { when } j=n+r\end{cases}
$$

where $1 / 2<\delta \leq 1$ is chosen so that all tuples $\left(\alpha_{j}^{r}\right)_{j=n+2}^{M}$ are in $\Lambda$. Further let

$$
\alpha_{j}^{M-n}= \begin{cases}0 & \text { if } j>n+3 \\ a & \text { if } j=n+2 \\ b & \text { if } j=n+3\end{cases}
$$

where $a, b \in[-1,1]^{2}$ are such that $\left(\alpha_{j}^{M-n+1}\right)_{j=n+2}^{M} \in \Lambda$. Denote by $\Lambda^{\prime}$ the set of all admissable pairs $(a, b)$. Notice that $\Lambda^{\prime} \subset[-1,1]^{2}$ has full measure. 
Now we can consider a system of $(M-n)$ equations with $(M-1)$ variables, each of the form (12) with coefficients determined by the above defined $(M-n-1)$-tuples $\left(\alpha_{j}^{r}\right)_{j=n+2}^{M}$ for $r=1, \ldots, M-n$. That is, we consider a system of equations with the matrix $A$ whose $r$-th row is given by

$$
\begin{aligned}
{\left[A_{r}\right]=\left[-\operatorname{sgn}\left(f_{1, n+r}\right) \varphi^{\prime}\left(\frac{\delta\left|f_{1, n+r}\right|}{L}\right), \ldots,-\operatorname{sgn}\left(f_{n, n+r}\right) \varphi^{\prime}\left(\frac{\delta\left|f_{n, n+r}\right|}{L}\right),\right.} & \underbrace{0, \ldots, 0}_{(r-1)}, \varphi^{\prime}\left(\frac{\delta}{L}\right), 0, \ldots, 0],
\end{aligned}
$$

when $1 \leq r \leq M-n-1$, and

$$
\begin{aligned}
{\left[A_{M-n}\right]=[-} & \operatorname{sgn}\left(a f_{1, n+2}+b f_{1, n+3}\right) \varphi^{\prime}\left(\frac{\left|a f_{1, n+2}+b f_{1, n+3}\right|}{L}\right), \ldots, \\
& -\operatorname{sgn}\left(a f_{n, n+2}+b f_{n, n+3}\right) \varphi^{\prime}\left(\frac{\left|a f_{n, n+2}+b f_{n, n+3}\right|}{L}\right), \operatorname{sgn}(a) \varphi^{\prime}\left(\frac{|a|}{L}\right), \\
& \left.\operatorname{sgn}(b) \varphi^{\prime}\left(\frac{|b|}{L}\right), 0, \ldots, 0\right] .
\end{aligned}
$$

Clearly rank $A \geq M-n-1$. Also, since $\left.u_{1}\right|_{S}, \ldots,\left.u_{n}\right|_{S}$ are linearly independent solutions, the solution space of $A$ has dimension greater or equal than $n$. Thus rank $A \leq M-n-1$.

Hence $\operatorname{rank} A=M-n-1$. Since rows $\left[A_{r}\right]$ for $1 \leq r \leq M-n-1$ are clearly linearly independent, hence the last row of $A$ is the linear combination of the first $(M-n-1)$ rows. Therefore, for all $(a, b) \in \Lambda^{\prime}$ we have

$$
\begin{aligned}
& \frac{\operatorname{sgn}(a) \varphi^{\prime}\left(\frac{|a|}{L}\right)}{\varphi^{\prime}\left(\frac{\delta}{L}\right)}\left(-\operatorname{sgn}\left(f_{1, n+2}\right) \varphi^{\prime}\left(\frac{\delta\left|f_{1, n+2}\right|}{L}\right)\right)+ \\
& +\frac{\operatorname{sgn}(b) \varphi^{\prime}\left(\frac{|b|}{L}\right)}{\varphi^{\prime}\left(\frac{\delta}{L}\right)}\left(-\operatorname{sgn}\left(f_{1, n+3}\right) \varphi^{\prime}\left(\frac{\delta\left|f_{1, n+3}\right|}{L}\right)\right)= \\
& =-\operatorname{sgn}\left(a f_{1, n+2}+b f_{1, n+3}\right) \varphi^{\prime}\left(\frac{\left|a f_{1, n+2}+b f_{1, n+3}\right|}{L}\right)
\end{aligned}
$$

In particular, when $b$ approaches 0 (and respectively when $a$ approaches 0 ) we obtain that $\lim _{t \rightarrow 0} \varphi^{\prime}(t)=0$ and

$$
\begin{aligned}
& \frac{-\operatorname{sgn}\left(a f_{1, n+2}\right) \varphi^{\prime}\left(\frac{|a|}{L}\right)}{\varphi^{\prime}\left(\frac{\delta}{L}\right)} \varphi^{\prime}\left(\frac{\delta\left|f_{1, n+2}\right|}{L}\right)=-\operatorname{sgn}\left(a f_{1, n+2}\right) \varphi^{\prime}\left(\frac{\left|a f_{1, n+2}\right|}{L}\right) \\
& \frac{-\operatorname{sgn}\left(b f_{1, n+3}\right) \varphi^{\prime}\left(\frac{|b|}{L}\right)}{\varphi^{\prime}\left(\frac{\delta}{L}\right)} \varphi^{\prime}\left(\frac{\delta\left|f_{1, n+3}\right|}{L}\right)=-\operatorname{sgn}\left(b f_{1, n+3}\right) \varphi^{\prime}\left(\frac{\left|b f_{1, n+3}\right|}{L}\right)
\end{aligned}
$$


Therefore, if $\operatorname{sgn}(a)=\operatorname{sgn}\left(f_{1, n+2}\right)$ and $\operatorname{sgn}(b)=\operatorname{sgn}\left(f_{1, n+3}\right)$ and if we denote

$$
x=\frac{a f_{1, n+2}}{L} \quad, \quad y=\frac{b f_{1, n+3}}{L}
$$

then (14), (15) and (13) imply that:

$$
\varphi^{\prime}(x+y)=\varphi^{\prime}(x)+\varphi^{\prime}(y)
$$

and (16) is valid for almost all $(x, y) \in\left[0, \frac{1}{L}\right] \times\left[0, \frac{1}{L}\right]$. Since $\varphi^{\prime}$ is increasing we conclude that $\varphi^{\prime}$ is linear on $\left[0, \frac{2}{L}\right]$ and thus there exists $C \geq 0$ so that

$$
\varphi(t)=C t^{2} \quad \text { for } \quad t \leq \frac{2}{L} .
$$

But then, if $C=0$ then there exists $t>0$ with $\varphi(t)=0$, or, if $C>0$ then $\varphi$ is similar to $t^{2}$, which contradicts our assumptions. Hence $f_{1, n+2}, f_{1, n+3}$ cannot both be nonzero.

The last part of Theorem 7 follows from the following result:

Theorem 10. (18, Theorem 6.1]) Let $\ell_{\phi}$ be a (real or complex) Orlicz space and let $x, y \in$ $\ell_{\phi}$, be disjoint elements such that $\|x\|_{\phi}=\|y\|_{\phi}=1$ and $\operatorname{span}\{x, y\}$ is 1 -complemented in $\ell_{\phi}$.

Then one of three possibilities holds:

(1) $\operatorname{card}(\operatorname{supp} x)<\infty$ and $\left|x_{i}\right|=\left|x_{j}\right|$ for all $i, j \in \operatorname{supp} x$; or

(2) there exists $p, 1 \leq p \leq \infty$, such that $\phi(t)=C t^{p}$ for all $t \leq\|x\|_{\infty}$; or

(3) there exists $p, 1 \leq p \leq \infty$, and constants $C_{1}, C_{2}, \gamma \geq 0$ such that $C_{2} t^{p} \leq \phi(t) \leq C_{1} t^{p}$ for all $t \leq\|x\|_{\infty}$ and such that, for all $j \in \operatorname{supp} x$,

$$
\left|x_{j}\right|=\gamma^{k(j)} \cdot\|x\|_{\infty}
$$

for some $k(j) \in \mathbb{Z}$.

\section{REFERENCES}

[1] M. Baronti and P. L. Papini. Norm-one projections onto subspaces of $\ell_{p}$. Annali di Mat. Pura ed Appl., 152:53-61, 1988.

[2] Y. Benyamini, P. Flinn, and D.R. Lewis. A space without 1-unconditional basis which is 1-complemented in a space with a 1-unconditional basis. Longhorn Notes, The University of Texas, Texas Funct. Anal. Seminar, pages 145-149, 1983-84.

[3] F. F. Bonsall and J. Duncan. Numerical ranges of operators on normed spaces and of elements of normed algebras, volume 2 of London Math. Soc. Lecture Notes. Cambridge Univ. Press, 1971.

[4] S. T. Chen, H. Hudzik, and A. Kamińska. Support functionals and smooth points in Orlicz function spaces equipped with the Orlicz norm. Math. Japon., 39:271-279, 1994. 
[5] E. W. Cheney and K. H. Price. Minimal projections. In A. Talbot, editor, Approximation Theory, Proceedings of a symposium held at Lancaster, July 1969, pages 261 - 289. Academic Press, 1970.

[6] I. Doust. Contractive projections on Lebesgue-Bochner spaces. In Function spaces (Proceedings of the Conference on Function Spaces in Edwardsville, IL held in May 1994), volume 172 of Lecture Notes in Pure and Appl. Math., pages 101-109. Dekker, New York, 1995.

[7] P. Flinn. On a theorem of N.J. Kalton and G.V. Wood concerning 1-complemented subspaces of spaces having an orthonormal basis. Longhorn Notes, The University of Texas, Texas Funct. Anal. Seminar, pages 135-144, 1983-84.

[8] R. Grzasślewicz and H. Hudzik. Smooth points of Orlicz spaces equipped with Luxemburg norm. Math. Nachr., 155:31-45, 1992.

[9] N. J. Kalton and B. Randrianantoanina. Surjective isometries of rearrangement-invariant spaces. Quart. J. Math. Oxford, 45:301-327, 1994.

[10] N. J. Kalton and G. V. Wood. Orthonormal systems in Banach spaces and their applications. Math. Proc. Camb. Phil. Soc., 79:493-510, 1976.

[11] D.R. Lewis. Ellipsoids defined by Banach ideal norms. Matematika, 26:18-29, 1979.

[12] J. Lindenstrauss. A remark on symmetric spaces. Israel J. Math, 13:317-320, 1972.

[13] J. Lindenstrauss and L. Tzafriri. Classical Banach spaces, Vol. 1, Sequence spaces. Springer-Verlag, Berlin-Heidelberg-New York, 1978.

[14] J. Lindenstrauss and L. Tzafriri. Classical Banach spaces, Vol. 2, Function spaces. Springer-Verlag, Berlin-Heidelberg-New York, 1979.

[15] G. Lumer. Semi-inner product spaces. Trans. Amer. Math. Soc., 100:29-43, 1961.

[16] G. Lumer. On the isometries of reflexive Orlicz spaces. Ann. Inst. Fourier, 13:99-109, 1963.

[17] G. Lumer and R. S. Phillips. Dissipative operators a Banach space. Pacific J. Math., 11:679-698, 1961.

[18] B. Randrianantoanina. 1-complemented subspaces of spaces with 1-unconditional bases. MSRI Preprint No. 1996-035.

[19] B. Randrianantoanina. Contractive projections and isometries in sequence spaces. to appear in Rocky Mountain J. Math.

[20] H. P. Rosenthal. Contractively complemented subspaces of Banach spaces with reverse monotone (transfinite) bases. Longhorn Notes, The University of Texas Functional Analysis Seminar, pages 1-14, 1984-5.

Department of Mathematics and Statistics, Miami University, Oxford, OH 45056

E-mail address: randrib@muohio.edu 